\title{
Genome-wide analysis discloses reversal of the hypoxia- induced changes of gene expression in colon cancer cells by zinc supplementation
}

\author{
Michal Sheffer ${ }^{1}$, Amos J. Simon ${ }^{2}$, Jasmine Jacob-Hirsch ${ }^{2}$, Gideon Rechavi ${ }^{2}$, Eytan \\ Domany ${ }^{1}$, David Givol ${ }^{3}$ and Gabriella D'Orazi ${ }^{4,5}$ \\ ${ }^{1}$ Department of Physics of Complex Systems, Weizmann Institute of Science, Rehovot 76100, Israel \\ 2 Department of Pediatric Hematology-Oncology, Chaim Sheba Medical Center and Sackler School of Medicine, Tel-Aviv \\ University, Tel-Aviv, Israel \\ ${ }^{3}$ Department of Molecular Cell Biology, Weizmann Institute of Science, Rehovot 76100, Israel \\ ${ }^{4}$ Department of Experimental Oncology, Molecular Oncogenesis Laboratory, National Cancer Institute Regina Elena; Rome, \\ Italy \\ ${ }^{5}$ Department of Oral Sciences, Nano and Biotechnology, University "G. d'Annunzio", Chieti, Italy \\ Correspondence to: David Givol, email: david.givol@weizmann.ac.il \\ Gabriella D'Orazi, email: gdorazi@unich.it
}

Keywords: hypoxia, cobalt, pathway analysis, Principal Component Analysis (PCA), cDNA microarray, colon cancer

Received: December 20,2011, Accepted: December 23, 2011, Published: December 24, 2011

Copyright: @ Sheffer et al. This is an open-access article distributed under the terms of the Creative Commons Attribution License, which permits unrestricted use, distribution, and reproduction in any medium, provided the original author and source are credited.

ABSTRACT:

Hypoxia-inducible factor 1 (HIF-1), the major transcription factor specifically activated during hypoxia, regulates genes involved in critical aspects of cancer biology, including angiogenesis, cell proliferation, glycolysis and invasion. The HIF1a subunit is stabilized by low oxygen, genetic alteration and cobaltous ions, and its over-expression correlates with drug resistance and increased cancer mortality in various cancer types, therefore representing an important anticancer target. Zinc supplementation has been shown to counteract the hypoxic phenotype in cancer cells, in vitro and in vivo, hence, understanding the molecular pathways modulated by zinc under hypoxia may provide the basis for reprogramming signalling pathways for anticancer therapy. Here we performed genome-wide analyses of colon cancer cells treated with combinations of cobalt, zinc and anticancer drug and evaluated the effect of zinc on gene expression patterns. Using Principal Component Analysis we found that zinc markedly reverted the cobalt-induced changes of gene expression, with reactivation of the drug-induced transcription of pro-apoptotic genes. We conclude that the hypoxia pathway is a potential therapeutic target addressed by zinc that also influences tumor cell response to anticancer drug.

\section{INTRODUCTION}

Hypoxia is a common state of cancer cells due to the lack of blood supply to the rapidly growing tumor. Hypoxia-inducible factor 1 (HIF-1) is the key factor that mediates adaptive response to hypoxia; it is an heterodimeric transcription factor consisting of the HIF-1b subunit, constitutively expressed in cells, and the HIF-1a subunit whose stability is enhanced by low intracellular oxygen and also by genetic alteration [1]. In normoxia, HIF-1a is hydroxylated by prolyl hydroxylases
(PHD1-3) at key proline residues in the oxygen-dependent degradation domain (ODD) facilitating interaction with the E3 ligase, Von Hippel-Lindau protein (VHL), which drives HIF-1a ubiquitination and degradation [2, 3]. Under hypoxic conditions, prolyl hydroxylation is inhibited, thereby stabilizing HIF-1a and EPAS1 (also known as HIF-2 $\alpha$ ), which can then translocate to the nucleus and bind to constitutively expressed HIF-1b forming the active HIF-1 complex [4]. In a screen from the National Toxicology Program [5], three out of 1408 tested compounds (o-phenantroline, IodoChlorohydroxiquinone 
Table 1: Hypoxia and cobalt treatment shared genes and their reversal by zinc supplementation. The table lists 54 upregulated and 12 down-regulated genes that are shared between the cobalt treatment (C) of RKO cells and the hypoxia modulated genes reported by at least two out of ten studies taken from the MSigDB [19]. The fold change of gene expression in RKO treated cells is shown for treatment by cobalt compared with untreated cells $(\mathrm{C}-0)$ and by treatment with zinc and cobalt compared with cobalt treated cells (ZCC); negative sign of the fold changes indicates decrease in expression.

\begin{tabular}{|c|c|c|c|c|}
\hline Symbol & Gene Title & C-0 & ZC-C & References \\
\hline Up-regulated & & Fold change & Fold change & \\
\hline P4HA1 & prolyl4-hydroxylase, alpha polypeptide I & 3.12 & -1.32 & $20-26,29$ \\
\hline ADM & adrenomedullin & 6.67 & -4.43 & $20-22,25-27$ \\
\hline BNIP3L & BCL2/adenovirus E1B 19kDa interacting protein 3-like & 4.51 & -2.09 & $20-23,25,26$ \\
\hline NDRG1 & $\mathrm{N}$-myc dow nstreamregulated 1 & 9.40 & -5.30 & $20,21,23,25,26,29$ \\
\hline SLC2A1 & solute carrier family 2 (facilitated glucose transporter), member 1 & 1.99 & -1.12 & $20-24,29$ \\
\hline AK3L1 & adenylate kinase 3 -like 1 & 1.75 & -1.29 & $21,23-25,29$ \\
\hline BHLHE40 & basic helix-loop-helix family, member e40 & 4.63 & -2.26 & $20,21,24-26$ \\
\hline C7orf68 & chromosome 7 open reading frame 68 & 3.00 & -2.25 & $20,21,23,26,29$ \\
\hline CCNG2 & cyclin G2 & 2.01 & -2.30 & $20-22,24,25$ \\
\hline ENO2 & enolase 2 (gamma, neuronal) & 3.90 & -2.34 & $20,21,23,25,26$ \\
\hline KDM3A & lysine (K)-specific demethylase $3 \mathrm{~A}$ & 3.98 & -1.88 & $20,21,24,25,26$ \\
\hline P4HA2 & prolyl4-hydroxylase, alpha polypeptide II & 1.82 & -1.55 & $20,21,24,25,26$ \\
\hline PGK1 & phosphoglycerate kinase 1 & 1.54 & -1.13 & $20-22,26,29$ \\
\hline CA9 & carbonic anhydrase IX & 7.60 & -1.35 & $20-22,29$ \\
\hline EGLN1 & egl nine homolog 1 (C. elegans) & 2.90 & -1.75 & $20,21,25,26$ \\
\hline GBE1 & glucan (1,4-alpha-), branching enzyme 1 & 3.17 & -1.84 & $20,21,25,26$ \\
\hline HK2 & hexokinase 2 & 1.85 & -1.17 & $20-22,26$ \\
\hline PFKFB4 & 6-phosphofructo-2-kinase/fructose-2,6-biphosphatase 4 & 16.27 & -4.59 & $20,24,26,29$ \\
\hline ALDOA & aldolase $\mathrm{A}$, fructose-bisphosphate & 1.74 & -1.32 & $22,24,29$ \\
\hline ALDOC & aldolase $\mathrm{C}$, fructose-bisphosphate & 6.93 & -3.57 & $21,23,26$ \\
\hline ANG & angiogenin, ribonuclease, RNase $\mathrm{A}$ family, 5 & 1.61 & -1.79 & $21,26,27$ \\
\hline ANKRD37 & ankyrin repeat domain 37 & 5.43 & -3.73 & $20,26,29$ \\
\hline HMOX1 & heme oxygenase (decycling) 1 & 6.47 & -2.87 & $22,23,28$ \\
\hline INSIG2 & insulin induced gene 2 & 2.57 & -2.11 & $20,21,26$ \\
\hline MAFF & v-maf musculoaponeurotic fibrosarcoma oncogene homolog $F$ (avian) & 1.87 & -1.48 & $21,25,26$ \\
\hline PDK1 & pyruvate dehydrogenase kinase, isozyme 1 & 2.56 & -1.18 & $20,21,26$ \\
\hline PFKFB3 & 6-phosphofructo-2-kinase/fructose-2,6-biphosphatase 3 & 1.55 & -1.41 & $20,21,26$ \\
\hline PGM1 & phosphoglucomutase 1 & 1.97 & -1.41 & $21,23,25$ \\
\hline SPAG4 & sperm associated antigen 4 & 2.88 & -1.19 & $21,25,26$ \\
\hline TMEM45A & transmembrane protein $45 \mathrm{~A}$ & 5.45 & -2.53 & $20,21,25$ \\
\hline ZNF292 & zinc finger protein 292 & 2.69 & -1.56 & $20,21,25$ \\
\hline $\mathrm{ABCB} 6$ & ATP-binding cassette, sub-family B (MDR/TAP), member 6 & 2.73 & -1.90 & 20,26 \\
\hline CSRP2 & cysteine and glycine-rich protein 2 & 2.10 & -2.05 & 20,21 \\
\hline FOS & FBJ murine osteosarcoma viral oncogene homolog & 2.99 & -1.83 & 21,22 \\
\hline GYS1 & glycogen synthase 1 (muscle) & 1.62 & -1.27 & 20,21 \\
\hline HCFC1R1 & host cell factor $\mathrm{C} 1$ regulator 1 (XPO1 dependent) & 1.69 & -1.87 & 21,26 \\
\hline KDMAB & lysine (K)-specific demethylase 4B & 2.21 & -1.82 & 21,26 \\
\hline LIMCH1 & LIM and calponin homology domains 1 & 1.99 & -1.46 & 21,25 \\
\hline RAB20 & RAB20, member RAS oncogene family & 3.88 & -2.20 & 20,23 \\
\hline RBPJ & recombination signal binding protein for immunoglobulin kappa $\mathrm{J}$ region & 1.58 & -1.36 & 21,26 \\
\hline RIOK3 & RIO kinase 3 (yeast) & 1.61 & -1.32 & 20,26 \\
\hline RORA & RAR-related orphan receptor $A$ & 3.98 & -2.79 & 20,26 \\
\hline SAP30 & Sin $3 \mathrm{~A}$-associated protein, $30 \mathrm{kDa}$ & 1.77 & -1.72 & 20,24 \\
\hline SCD & stearoyl-CoA desaturase (delta-9-desaturase) & 1.72 & -1.67 & 23,26 \\
\hline SERTAD2 & SERTA domain containing 2 & 1.66 & -1.57 & 20,23 \\
\hline SLC16A3 & solute carrier family 16 , member 3 (monocarboxylic acid transporter 4 ) & 1.57 & -1.27 & 24,28 \\
\hline STBD1 & starch binding domain 1 & 1.58 & -1.75 & 21,23 \\
\hline WSB1 & WD repeat and SOCS box-containing 1 & 1.79 & -1.29 & 20,21 \\
\hline YEATS2 & YEATS domain containing 2 & 1.58 & -1.26 & 20,21 \\
\hline SPOCK1 & sparc/osteonectin, cw cv and kazal-like domains proteoglycan (testican) 1 & 1.57 & -1.37 & 21,25 \\
\hline \multicolumn{5}{|l|}{ Down-regulated } \\
\hline EEF1E1 & eukaryotic translation elongation factor 1 epsilon 1 & -1.68 & -1.01 & $20,21,25$ \\
\hline BOP1 & block of proliferation 1 & -1.68 & 1.46 & 21,25 \\
\hline $\mathrm{DDX} 21$ & DEAD (Asp-Glu-Ala-Asp) box polypeptide 21 & -1.61 & 1.56 & 20,25 \\
\hline IL18R1 & interleukin 18 receptor 1 & -1.95 & 1.97 & 20,28 \\
\hline NIP7 & nuclear import 7 homolog (S. cerevisiae) & -1.58 & 1.24 & 20,21 \\
\hline RANGAP1 & Ran GTPase activating protein 1 & -1.55 & 1.29 & 20,21 \\
\hline RRP15 & ribosomal RNA processing 15 homolog (S. cerevisiae) & -1.65 & 1.36 & 21,25 \\
\hline RRS1 & RRS1 ribosome biogenesis regulator homolog (S. cerevisiae) & -1.59 & 1.56 & 21,25 \\
\hline RUVBL1 & RuvB-like 1 (E. coli) & -1.80 & 1.53 & 20,25 \\
\hline SLC25A15 & solute carrier family 25 (mitochondrial carrier; ornithine transporter) member 15 & -1.75 & 1.33 & 20,25 \\
\hline SRM & spermidine synthase & -1.55 & 1.48 & 21,25 \\
\hline WDR4 & WD repeat domain 4 & -1.51 & 1.65 & 20,25 \\
\hline
\end{tabular}


and cobalt sulfate) were selected as hypoxia-mimetic whereas cobalt was the only one that interacted with HIF-1 in a manner similar to hypoxia. Thus, cobaltous ions have been shown to inhibit hydroxylation of HIF1a and therefore induce elevated HIF-1a protein levels,

Table 2: Schematic representation of the several combination treatments between ADR, cobalt and zinc performed for microarray analysis. Each sample was represented by duplicates.

\begin{tabular}{|c|c|c|c|c|}
\hline Samples & Abbreviation & ADR & Zinc & Cobalt \\
\hline Untreated & $(0)$ & - & - & - \\
\hline ADR & $(\mathrm{A})$ & + & - & - \\
\hline Zinc & $(\mathrm{Z})$ & - & + & - \\
\hline Cobalt & $(\mathrm{C})$ & - & - & + \\
\hline ADR+ Zinc & $(\mathrm{AZ})$ & + & + & - \\
\hline ADR+ Cobalt & $(\mathrm{AC})$ & + & - & + \\
\hline Zinc+ Cobalt & $(\mathrm{ZC})$ & - & + & + \\
\hline ADR+ Zinc+ Cobalt & $(\mathrm{AZC})$ & + & + & + \\
\hline
\end{tabular}

mimicking hypoxia [1]. The interest in HIF-1 comes form the fact that HIF-1 controls the expression of numerous genes involved in many aspects of cancer progression, including angiogenesis (e.g., vascular endothelial growth factor - VEGF), metabolic adaptation (e.g., Glut1), apoptosis resistance (e.g., Bcl2), invasion and metastasis (e.g., c-Met) [6]. The levels of HIF-1a subunit are often increased in most solid human tumors including colon, brain, breast, gastric, lung, skin, ovarian, prostate, renal, and pancreatic carcinoma, rendering tumor cells resistant to conventional chemotherapy and selecting a more malignant and invasive phenotype leading to poor prognosis [6]. Inhibition of HIF-1a may, therefore, represent an attractive strategy with potential for synergism with other antitumor therapies [7, 8].

We found that zinc supplementation to highly invasive and angiogenic glioblastoma cells or to prostate cancer cells, either constitutively hypoxic or after induction of hypoxia, downregulates HIF-1a protein levels and

$\mathbf{A}$

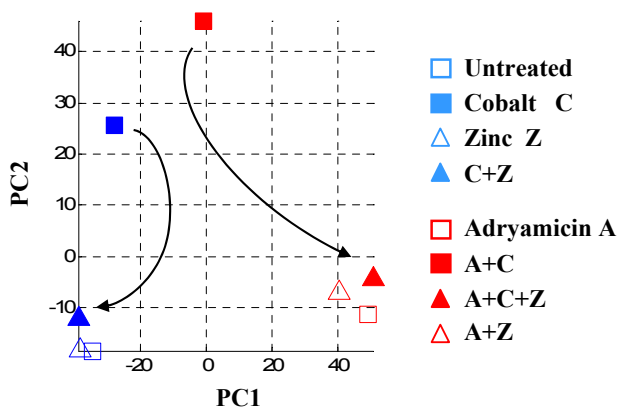

B

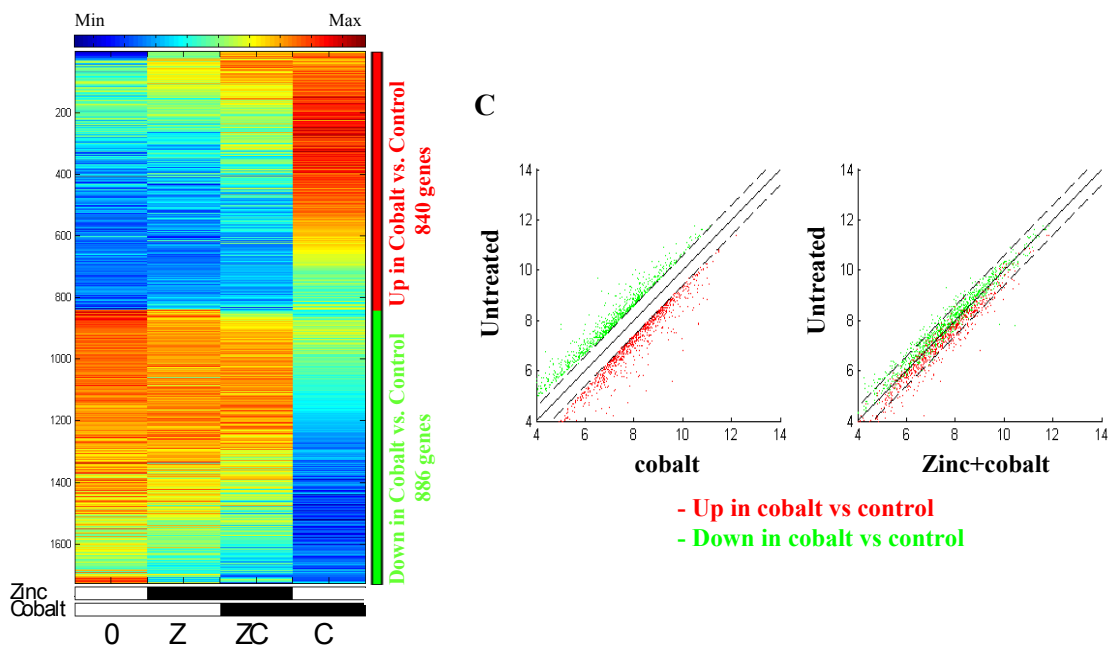

Figure 1: Expression of genes regulated by cobalt and reversed by zinc. (A). PCA of the samples, after log2 transformation and averaging between replicates, in the space of all probesets. Filled markers represent cobalt, red markers represent ADR and the triangular markers represent zinc. The arrows indicate the reverse effect of the zinc treatment when applied on cobalt, returning the expression to the original (untreated) position. (B) Heatmap of the genes that were differentially expressed (passed 5\% FDR, and had at least 1.5 fold change) between cobalt treated cells (C) and untreated cells (0). Data is centered and normalized after log2 and averaging of replicates, sorted using SPIN [51]. The red sidebar represents up-regulated genes and the blue sidebar represents down-regulated genes. (C) Left panel. Scatter plots of the genes that were up regulated (in red) and down regulated (in green) by cobalt (C), compared with untreated cells (0). Dashed lines represent represent 1.5 fold change ( 0.585 in $\log 2$ scale). Right panel. The same genes when comparing zinc + cobalt (ZC) to untreated cells (0), showing the reversal of gene expression by supplemental zinc on cobalt treated cells. 
inhibits HIF-1 activity, resulting in the inhibition of VEGF expression, angiogenesis and tumor cell invasiveness [9]. This is relevant in light of recent findings showing that treatment of cancer by anti-angiogenic agents like anti VEGF or small molecule inhibitors of tyrosine kinase may result in hypoxia that selects for more malignant

A

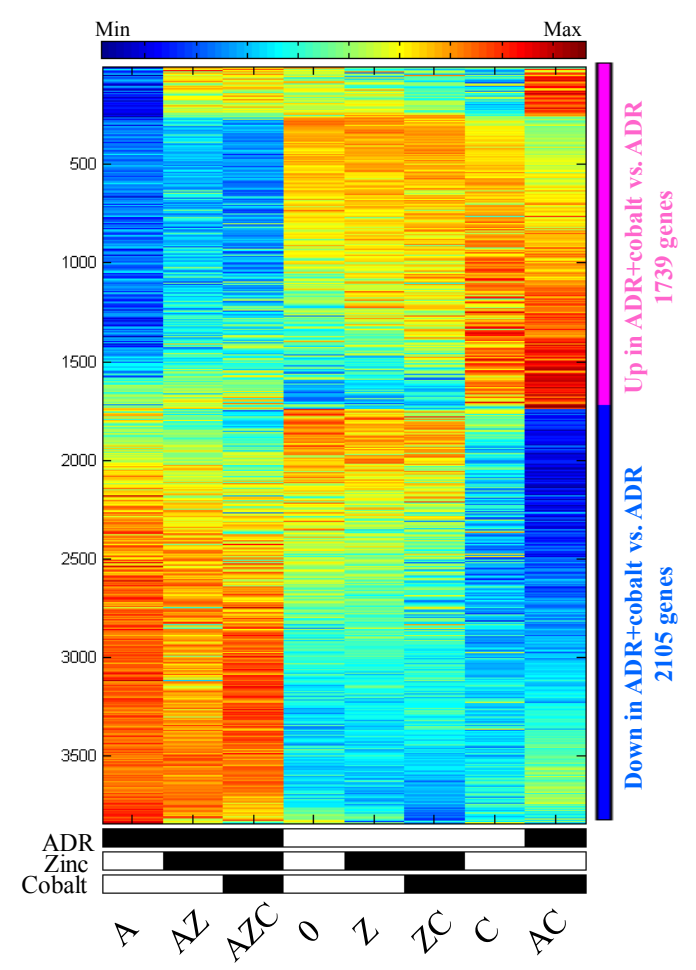

B

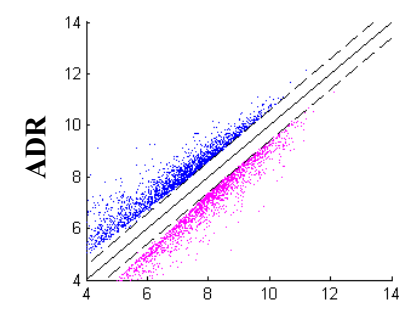

ADR+cobalt

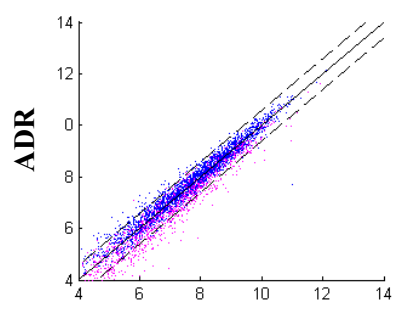

ADR+zinc + cobalt
- Up in ADR+cobalt vs ADR

- Down in ADR+cobalt vs ADR

Figure 2: Expression of genes regulated by ADR+cobalt and reversed by zinc. (A) Heatmap of the genes that were differentially expressed (passed 5\% FDR, and had at least 1.5 fold change) between ADR+cobalt treated cells (AC) and ADR treated cells (A). Data is centered and normalized after $\log 2$ and averaging of replicates, sorted using SPIN. The pink sidebar represents up-regulated genes and the blue sidebar represents down-regulated genes. (B) Left panel. Scatter plots of the genes that were up regulated (in pink) and down regulated (in blue) by $\mathrm{ADR}+$ cobalt (AC), compared with ADR treated cells (A). Dashed lines represent 1.5 fold changes (0.585 in $\log _{2}$ scale). Right panel. The same genes in the comparison of $\mathrm{ADR}+$ zinc+cobalt $(\mathrm{AZC})$ to ADR treated cells (A), showing the counter effect of zinc on cobalt treated cells. metastatic and invasive cells, that eventually lead the tumors to relapse as a more invasive and metastatic disease $[10,11]$. Similar results may apply also for the treatment with antibodies when the signaling triggered by the antibody affects the HIF-1 pathway [12]. Zinc is a trace element that is essential for the normal function of cells and is a cofactor for a wide range of structural proteins, enzymes, and transcription factors involved in key cellular functions such as the response to oxidative stress, DNA damage repair, cell cycle progression and apoptosis [13]. The mechanisms through which gene expression is regulated by zinc are central to cellular homeostasis, thus, zinc is an essential prerequisite for the execution of many signaling pathways in eukaryotes [14]. Preclinical studies have shown that zinc exerts a positive beneficial effect against chemically induced pre-neoplastic progression in rats and provides an effective dietary chemopreventive approach to disease [15]. On the other hand, zinc deficiency has been associated with induction of cancer [16]. Furthermore, in vitro and in vivo studies by our group have shown that zinc supplementation to cancer cells improves the chemotherapeutic response with reactivation, for instance, of inactive onco-suppressor p53 and apoptosis $[17,18]$. Understanding the molecular pathways modulated by zinc under hypoxia may provide the basis for reprogramming signalling pathways for anticancer therapy and hopefully improve classical anticancer therapies. To this aim, we performed a genomewide expression analysis in colon cancer cells treated with different combinations of hypoxia-mimetic cobalt, zinc and anticancer drug. Our strategy identified differences in gene expression among the combination treatments. The most remarkable result was that zinc reversed gene expression of most genes modulated by hypoxia, including genes involved in metabolism, proteasomal build-up, and amino acid biosynthesis. As a result of hypoxic phenotype reversion, zinc supplementation restored the drug-induced apoptosis, inhibited by hypoxia. Our studies suggest that zinc supplementation to cancer cells may have an effective anticancer outcome by targeting the hypoxia pathway and therefore provide the molecular basis for the combination treatment of tumors by zinc with classical anti-tumoral drugs.

\section{RESULTS}

\section{Expression of the modulated genes shared between cobalt and hypoxia}

Low oxygen as well as cobaltous ions inhibit hydroxylation of HIF-1a and therefore induce elevated HIF-1a protein levels, mimicking hypoxia [1]. Here, we first attempted to evaluate the extent of similarity in gene expression between cobalt and hypoxia treatment by 
constructing a list of hypoxia genes using hypoxia related gene sets that were published on the MSigDB database [19 by ten different studies [20-29]. This resulted in 150 up-regulated (hypoxia up) and 76 down-regulated (hypoxia down) genes that appeared in at least two out of the ten hypoxia studies (data not shown). When the 150 and 76 modulated genes were intersected with the modulated genes in the cobalt (C) treatment of RKO cells (Supplementary Table S1), the resulting shared genes were found to be 54 out of the 150 'hypoxia up', and 12 out of the 76 'hypoxia down' genes (Table 1, column C-0). This significant overlap is in agreement with many studies on hypoxia-like effect by cobalt, showing high level of similarity in modulated genes between hypoxia and cobalt treatment $[30,31]$.

Among the shared 'hypoxia up' genes we identified genes involved in carbohydrates metabolism, fructose, mannose, and glycolysis, (i.e., SLC2A1, also known as GLUT1, PGM1, ALDOA, ALDOC, PFKFB3, PFKFB4, GYS1, GBE1, HK2, ENO2 and PGK1), genes involved in oxidoreductase activity (i.e., SCD, P4HA2, P4HA1,
HMOX1 and EGLN1), in autophagy and tumor cell survival (i.e., BNIP3L) [32], in $\mathrm{pH}$ regulation (i.e., CA9) [33] in multidrug resistance (i.e., ABCB6) [34] in cell survival and proliferation (i.e., ADM, cyclin G2), in angiogenesis (i.e., EGLN1, ANG and ANGPTL4). We also found newly identified HIF-1a target genes such as TMEM45A, ANKRD37 and WSB1 [35], the latter one being involved in ubiquitination and degradation of HIPK2 [36], a putative tumor suppressor and p53 apoptotic regulator [37] that is down-regulated in hypoxia [38], supporting the hypoxia-mimetic function of cobalt.

Since we recently showed that the hypoxic phenotype can be inhibited by zinc supplementation to cancer cells $[9,17]$, we next evaluated the effect of zinc treatment on the cobalt modulated genes. Interestingly, we found that zinc markedly reverted the differential expression of genes shared between hypoxia and cobalt (Table 1, column ZC-C), in support of our biological results [9, 17]. Although some of the up- and down-regulated genes were reversed by less than 1.5 fold change, the expression levels of most of the 'hypoxia up' genes (34 out of 54) and

Table 3: Pathways that were found to be enriched with genes modulated by zinc in AZC-AC and A-0. Enrichment analysis of the cluster genes shown in the heathmaps in Fig. 3A (pink and green groups) (see Methods). The arrows indicate the up- and downregulated genes. Numbers in parenthesis indicate the number of modulated gene.

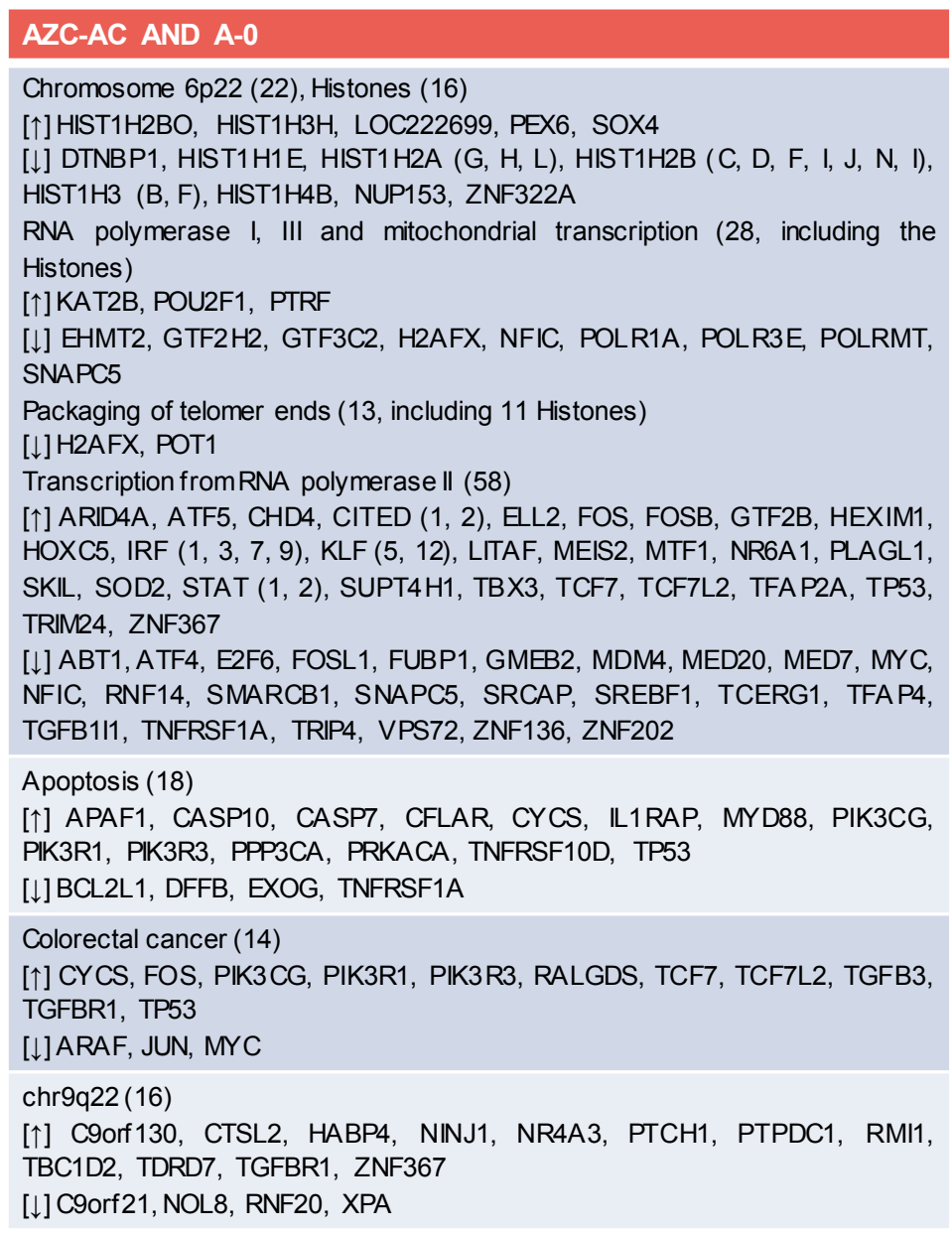


5 of the 12 'hypoxia down' genes were reversed by zinc supplementation to cobalt treatment by more than 1.5 fold change (Table 1).

\section{Zinc supplementation reverses the gene expression pattern induced by cobalt}

We next compared global gene expression variation between samples treated with different combination of cobalt (C), zinc (Z) and ADR (A), as shown in Table 2. The number of genes modulated by each treatment is shown in Supplementary Table S1. We used Principal Component Analysis (PCA), a method that reveals the internal structure of high dimensional data in a way which best explains the variance in the data [39]. PC1, the first principal component, shows that ADR treatment had the strongest effect on the cells (Fig. 1A), as PC1 separates the samples into two groups according to the ADR effect, differentiating between samples treated with ADR (red) and without ADR (blue). On the other hand, PC2 separates the cobalt and ADR+cobalt samples (filled red and blue squares) from the rest of the samples
(Fig. 1A). Interestingly, zinc treatment shifted the cobalt sample to the untreated and zinc-treated samples (Fig. 1A, see arrow) and the ADR+cobalt sample to the ADR and ADR+zinc samples (Fig. 1A, see arrow), suggesting that zinc counteracts and reverses the effect of cobalt on gene expression. Indeed, this reversal effect was also evident in heatmaps of the normalized expression data, showing that most genes up-regulated by cobalt, marked by the red sidebar, were down-regulated after zinc treatment, while the group of genes down-regulated by cobalt, marked by the green sidebar, was up-regulated after zinc treatment (Figure 1B, compare $\mathrm{C}$ with $\mathrm{ZC}$ column). This expression reversal was further analysed in the scatter plots showing the same red and green groups of genes comparing cobalt treatment with the untreated sample (Fig. 1C, left panel). The right scatter plot in Fig. $1 \mathrm{C}$ shows the same groups when comparing cobalt+zinc with the untreated sample. In this comparison, almost all the genes are within the 1.5 fold change range (marked by dashed lines), meaning that their expression levels were shifted towards their original (untreated) values.

The strong effect of zinc on cobalt-induced gene expression was evident in the samples treated with ADR.

Table 4: Pathways that were found to be enriched with genes modulated by zinc in both AZC-AC and ZC-C. Enrichment analysis of the cluster genes shown in the heathmaps in Fig. 3A (light blue and violet groups) (see Methods). The arrows indicate the up- and down-regulated genes. Numbers in parenthesis indicate the number of modulated gene.

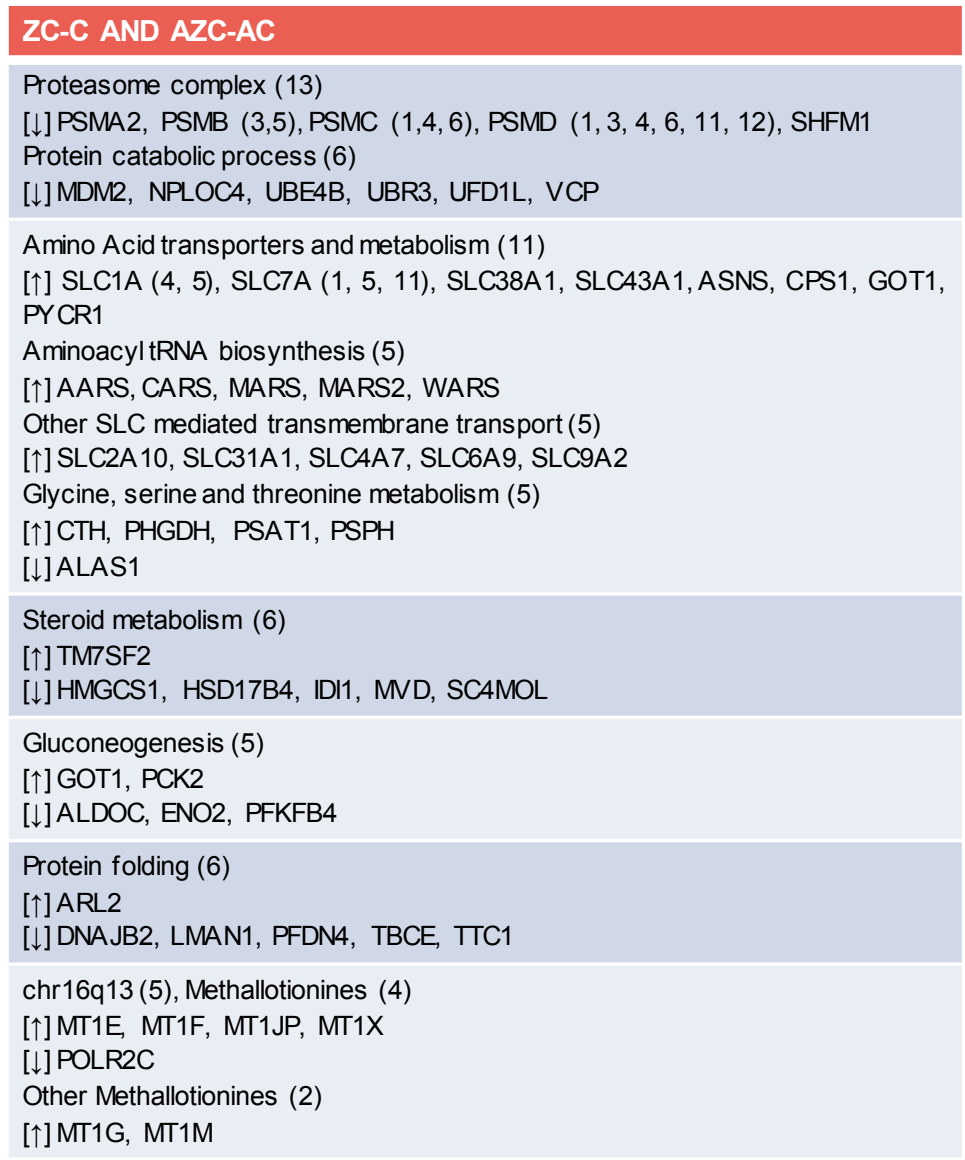


The heatmap shows that cobalt treatment inhibited the effect of ADR on gene expression (Fig. 2A, compare column A with AC). Interestingly, zinc treatment counteracted the effect of cobalt (Fig. 2A, compare column AC with AZC), restoring the ADR-induced gene expression (Fig. 2A, compare column AZC with A). Of note, zinc treatment per se did not change the ADR- induced gene expression (Fig. 2A, compare column A with AZ), suggesting that the main variation obtained was by cobalt treatment. This expression reversal was also apparent in the scatter plots showing the comparison of AC with A (Fig. 2B, left panel), and then reversal of expression changes in AZC compared with A (Fig. 2B, right panel), showing that zinc supplementation to $\mathrm{AC}$

Figure 3: Zinc and adriamycin regulated genes in cobalt treated cells. (A). Venn diagram of the up- and down-regulated genes (passed 5\% FDR, and had at least 1.5 fold change), following zinc supplement on cobalt treated (ZC) compared with cobalt treated cells (C), zinc supplement to ADR+cobalt treated cells (AZC) compared with ADR+cobalt treated cells (AC), and when comparing ADR treated cells (A) with untreated cells (0). The light blue and violet groups represent genes that are shared between AZC-AC and ZC-C (246 upregulated and 198 down-regulated genes, respectively). The pink and green groups represent genes that are shared between AZC-AC and A-0, not including ZC-C (956 up-regulated and 784 down-regulated genes, respectively). The intersection of all three comparisons (i.e. AZC-AC and ZC-C and A-0) contains 28 up regulated and 31 down regulated genes. (B). Heatmap of the genes that belong to the colored groups (see Venn diagram, sidebar). Expression values are centered and normalized after $\log 2$ and averaging of replicates, sorted using SPIN. (C). Heatmap of apoptotic genes that were regulated by zinc addition to ADR+cobat treated cells. Expression values are presented as $\log 2$ fold change compared with the untreated cells. The differences between AZC column and AC column are at least 1.5 fold changes. The up-regulated genes belong to the pink group and the down-regulated genes belong to the green group.

A
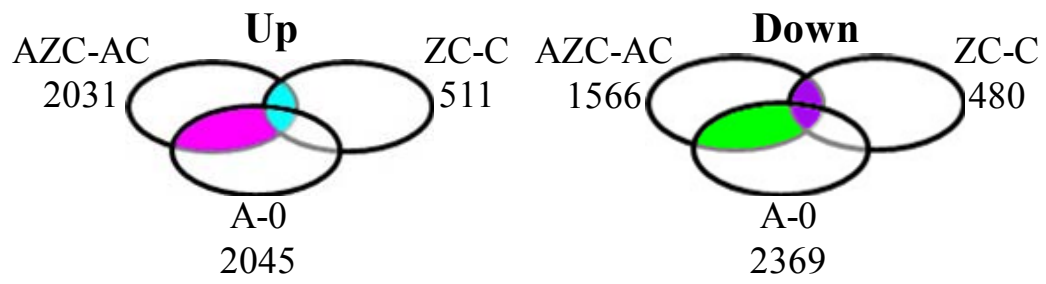

\section{B}

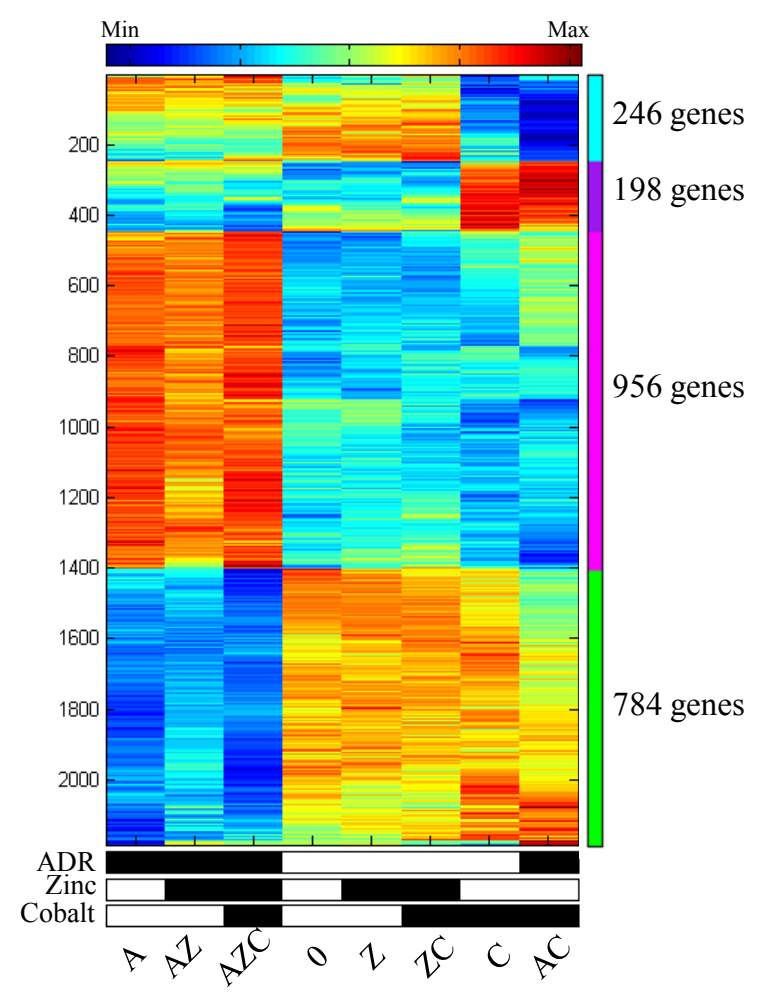

C

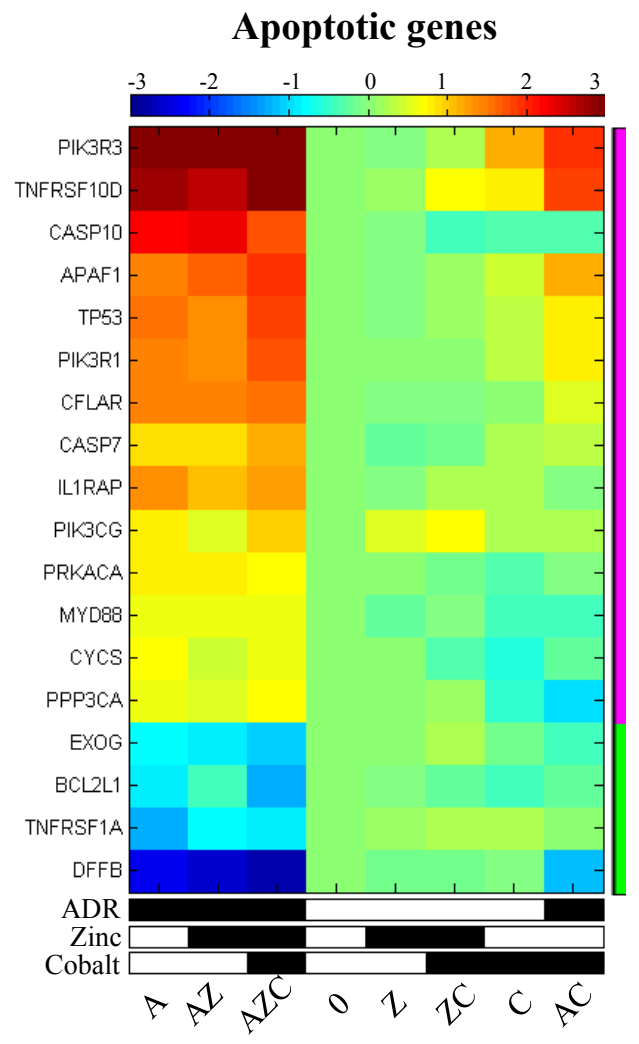


antagonized the effect of cobalt and decreased expression of the up-regulated genes towards their expression levels in A cells. The same applies to the down regulated genes. Altogether, these findings demonstrate that most of the genes that were significantly modulated by cobalt, when compared with the untreated cells, were reversed by zinc almost to their previous expression.

\section{Zinc restores the ADR-induced pro-apoptotic pathway in the presence of cobalt}

The next step in our analysis was the identification of the cellular pathways that are enriched within groups of modulated genes by the different treatments (see Methods). We first analysed the set of genes modulated in the comparison of AZC with AC treatments (denoted by AZC-AC in Supplementary Table S1). The result is shown in the Venn diagrams and includes 956 up-regulated genes (Fig. 3A, left panel, pink) and 784 down regulated genes (Fig. 3A, right panel, green). Most likely, the genes of these groups, which intersected with genes modulated in the A-0 comparison but not with ZC-C comparison, are good candidates to include genes for apoptotic response. Another group of genes that are modulated by zinc either in the presence or in the absence of ADR, is represented by the intersection of $\mathrm{AZC}-\mathrm{AC}$ with $\mathrm{ZC}-\mathrm{C}$, and may not contribute to the chemosensitivity of the cells, since cells treated with zinc+cobalt do not undergo apoptosis [17]. This intersection includes 246 up-regulated genes (Fig. 3A, left panel, light blue) and 198 down-regulated genes (Fig. 3A, right panel, violet). The normalized expression levels of the genes that belong to these two groups are shown in the heatmap using the same colors than the Venn diagram, indicated on the side bar (Fig. 3B). The first two clusters of genes (light blue, violet) showed differential expression when comparing their expression in the cobalt treated samples $(\mathrm{AC}, \mathrm{C})$ versus the other samples. The third and fourth clusters (pink, green) showed differential expression when comparing the ADR related samples (A, AZ, AZC) with the rest of the samples. The reversal by zinc supplementation to cobalt treated cells in the pink and green clusters occurred only in the presence of ADR (Fig. 3B, AZC vs. AC). In order to determine putatively affected pathways, enrichment analysis was performed on these clusters (see Methods). The first enrichment analysis was performed on genes belonging to the pink and green clusters (Fig. 3A, 3B). We found that the apoptotic pathway showed one of the highest score for enrichment, comprising 18 genes between up-regulated pro-apoptotic genes (i.e., caspase 7 and 10, cytochrome C (CYCS) and the apoptotic protease-activating factor APAF1, etc) and down-regulated anti-apoptotic genes (i.e., inhibitor of cell death BCL2L1, etc.) (Table 3). The heatmaps of the normalized expression data show that the apoptotic genes, up-regulated by ADR (A), were downregulated by cobalt
(AC) but restored by zinc (AZC) (Fig. 3C). As expected, the apoptotic genes were neither up-regulated by zinc nor in zinc-cobalt treatment (Fig. 3C, ZC).

\section{Enrichment analyses of the cluster genes showing additional modified pathways}

In addition to the apoptosis pathway, Table 3 reflects the effect of ADR on chromatin and the clusters that contain these genes showed enrichment for genes involved in chromatin structure like histones, some of them involved with enzymes of ribosomal RNA synthesis like RNA polymerase I, and III. Many (over 50) of the genes in this group are involved in facilitating transcription of coding RNA by RNA polymerase II. In addition, genes in these clusters take part in different signaling pathways such as insulin receptor, PI3K, TP53, TGFB that are also related to colorectal cancer. The pathways enriched within the light blue and pink clusters (Fig. 3B) reflect the effect of zinc on cobalt treatment and are mainly involved in carbohydrates metabolism, in protein synthesis and in regulation of metallothioneins (Table 4). Protein translation is one of the processes down-regulated in hypoxia [40]. In this regard, we found increased activation by zinc of pathways, such as metabolism of amino acid, aminoacyl tRNA biosynthesis and transporters of amino acids that will result in increase of protein synthesis (Table 4). On the other hand, the genes that were downregulated by zinc are enriched in pathways of protein degradation and in proteolytic enzymes associated with protein degradation (Table 4). Decrease in the expression of these genes results in decrease of protein degradation in the cells, which complements the increase in protein synthesis.

\section{DISCUSSION}

Hypoxia is a common state within many growing tumors because of lack of adequate blood supply, that promotes cell survival and blocks cell death. Therefore, targeting hypoxia is an attractive strategy to inhibit tumor growth and reactivate drug response. In the present work we aimed at evaluating the global gene expression pattern of colon cancer cell line, treated with the hypoxiamimetic cobalt and the antagonistic effect of zinc on the gene expression level and particularly with regard to response to drug (ADR) treatment. As demonstrated here, zinc reverted almost completely the hypoxia-induced gene expression. This was markedly evident by using the Principal Component Analysis (PCA) [39], a method that reveals the internal structure of high dimensional data in a way which best explains the variance in the data. This method, clearly substantiated how the zinc treatment shifted the cobalt samples near the untreated or zinc-treated samples (see Fig. 1A, 1B). The positive 
effect of cobalt inhibition was then corroborated with drug treatment (see Fig. 1A, Fig. 2).

How could zinc down-regulate the hypoxia-induced gene expression? One mechanism could be through inhibition of HIF-1 activity that mediates adaptive responses to changes in tissue oxygenation. Thus, our previous findings showed that zinc supplementation to cancer cells downregulates $\mathrm{HIF} 1 \alpha / 2 \alpha$ protein levels with a mechanism that involves PHD and VHL, leading to inhibition of HIF-1 activity [9]. This was particularly interesting in vivo, where the zinc-inhibitory of HIF-1a was reached inside the xenograft tumor injected in nude mice, meaning that oral zinc administration is able to reach the tumor site and modify the intratumoral HIF-1a expression [9]. The specific effect of zinc on HIF-1 activity was evident here at the molecular level, where the list of genes up-regulated by cobalt treatment and then reversed to their initial expression values by zinc supplementation (see Table 2), largely reflects the HIF-1 target genes (i.e., SLC2A1, BNIP3L, CA9, HK2, ALDOC, TMEM45A, WSB1, etc.) $[6,35]$. A large overlap with hypoxia related genes compiled by MSigDB database [19] on the basis of ten different studies [20-29] supported the hypoxia-like effect of cobalt.

Is it possible to clinically exploit the zinc-induced HIF-1 inhibition in tumor treatments? It is well known that hypoxia leads to chemoresistance which is a significant obstacle to successful cancer treatment [6]. One of the mechanisms of apoptosis resistance could be through expression of GLUT-1 or glycolytic enzymes, e.g., Aldolase $A / C$ and hexokinases [41-43]. Besides these protective mechanisms that depend on glucose metabolism, alternative survival mechanisms that block apoptosis under hypoxia have been also implicated. These survival pathways may involve overexpression of anti-apoptotic proteins, e.g., Bcl-2 and inhibition or downregulation of pro-apoptotic proteins such as Bid or Bax [43]. Here, we found that zinc supplementation markedly reversed both the glycolytic pro-survival and anti-apoptotic pathways (see Table 1), leading to restoration of chemosensitivity under hypoxia. Thus, the ADR-induced gene expression, partially abrogated by the cobalt treatment, was restored by zinc supplementation almost to the original expression level of ADR (see Fig. 1A, Fig. 2), therefore giving a molecular answer to the initial question.

Recently, HIF-1 has been shown to antagonize the p53 apoptotic activity [44] and in particular we showed that this depends on HIF-1-induced HIPK2 downregulation [45]. A major determinant of a successful cancer therapy is the ability of cancer cells to activate apoptotic cell death, mainly through intact p53 function, and much experimental and pre-clinical effort is devoted to reactivation of inactive and/or mutant $\mathrm{p} 53$. Therefore, by assuming that a solid tumor presents intratumoral hypoxia, the zinc-induced HIF-1 inhibition should rescue the p53 function in tumors bearing wtp53 and
HIPK2. As a proof of principle, we found here that one of the ubiquitine ligases involved in HIPK2 degradation under hypoxia, that is WSB-1 $[35,36]$, was up-regulated by cobalt but downregulated by zinc supplementation, strongly supporting the role of zinc in reactivating the HIPK2/p53 oncosuppressor axis under hypoxia. Another molecule important in $\mathrm{p} 53$ regulation is the MDM2 oncogene that downregulates both $\mathrm{p} 53$ and HIPK2 protein levels, strongly impairing tumor treatments [46]. Here, we found that MDM2 was one of the genes that were downregulated by zinc (Table 4), supporting, at molecular level, the zinc inhibitory effect on MDM2 activity [17, 47].

Although much more effort must be taken to understand the physiological implications of the various pathways modified by hypoxia and/or cobalt and after zinc supplementation, our findings provide the molecular basis for the reversal of the hypoxia-induced changes of gene expression by zinc, which may have an effective anticancer outcome through a more efficient utilization of chemotherapy.

\section{MATERIALS AND METHODS}

\section{Cell lines and treatments}

RKO human colon carcinoma cells (wild-type p53) were maintained in RPMI-1640 (Life TechnologyInvitrogen) supplemented with $10 \%$ heat-inactivated fetal bovine serum plus glutamine and antibiotics in humidified atmosphere with $5 \% \mathrm{CO}_{2}$ at $37^{\circ} \mathrm{C}$. Subconfluent cells were seeded the day before the treatment. The cells were pre-treated for 16 hours with $\mathrm{CoCl}_{2}(200 \mu \mathrm{M})$ and $\mathrm{ZnCl}_{2}$ $(100 \mu \mathrm{M})$ before adding adryamicin (ADR) $(1.5 \mu \mathrm{M})$ for additional 8 hours. The list of the different combination treatments used for the analyses is shown in Table 2, each treatment was prepared in duplicates.

\section{RNA extraction and reverse transcription (RT)- PCR analysis}

After treatments, cells were harvested in TRIzol Reagent (Invitrogen) and total RNA was isolated following the manufacturer's instructions. The first strand cDNA and the semi-quantitative RT-PCRs were carried out essentially as described $[17,18]$. Part of the cDNA was used to validate the system through analyses of gene transcription by RT-PCR by using genes specific oligonucleotides under conditions of linear amplification (data not shown). Total RNA was hybridized to Affymetrix HU-gene st1.0 microarrays. 


\section{Data analysis}

For normalization of the data from the 28,830 probesets of the arrays, Iter-Plier [48] algorithm was used on the .cel files, followed by modified Lowess correction algorithm [49] and $\log 2$ transformation. The mean intensity of each probe set in each pair of replicates was calculated, producing 'averaged data' for nine different conditions. The histograms of these nine conditions are plotted in Supplementary Figure S1. Intensity dependent variance was estimated for each probe set, using the distribution of the differences between repeats of probe sets having similar mean intensity [50]. To look for differentially expressed probe sets between the different conditions, 17 comparisons were made (described in Supplementary Table S1), Each comparison was made between two corresponding pairs of replicates, using a filtering step which included probe sets that were above threshold ( $\mathrm{t}=4)$ in both replicates, in at least one of the conditions. FDR of $5 \%$ was applied on all probe sets to control the number of false positives. An additional filter was imposed, requiring the fold change of the average gene expression to exceed 1.5. Unique gene representation of the data (known gene symbols only) was calculated for each gene symbol by averaging over all probe sets of that gene, and by averaging the replicates of each treatment.

To extract some biological meaning from differentially expressed genes, we looked at gene sets that share a common biological function such as cellular pathways. In order to do this we intersected the list of co-expressed genes with a subset of gene sets from the MsigDB [19] dataset, that belong to the positional, canonical pathways and GO biological process collections, retaining gene sets of minimal size of 8 and maximal size of 500 genes. Each gene set was assigned an enrichment score using the hyper geometric test; sets that passed FDR of $10 \%$ and included at least 5 genes were considered as significantly enriched.

\section{ACKNOWLEDGEMENTS}

This work was supported by AIRC Grant (G.D.O), by Grant from the Henry J Leir Charitable Foundation (E.D. and M.S.), and by the Flight Attendants Medical Research Institute (G.R.). We thank Rosa Puca for technical help in the early stages of this study. G.R. holds the Djerassi Chair in Oncology at the Tel-Aviv University.

\section{REFERENCES}

1. Wang GL, Jiang BH, Rue EA, Semenza GL. Hypoxiainducible factor 1 is a basic-helix-loop-helix-PAS heterodimer regulated by cellular O2 tension. Proc Natl Acad Sci U S A. 1995; 92:5510-5514.

2. Ivan M, Kondo K, Yang H, Kim W, Valiando J, Ohh M,
Salic A, Asara JM, Lane WS, Kaelin WG Jr. HIFaplha targeted for VHL-mediated destruction by prolyl hydroxylation: implications for $\mathrm{O}_{2}$ sensing. Science. 2001; 292:464-468.

3. Jakkola P, Mole DR, Tian YM, Wilson MI, Gielbert J, Gaskell SJ, Kriegsheim Av, Hebestreit HF, Mukherji M, Schofield CJ, Maxwell PH, Pugh CW, Ratcliffe PJ. Targeting of HIF-1alpha to the von Hippel-Lindau ubiquitylation complex by $\mathrm{O}_{2}$-regulated prolyl hydroxylation. Science. 2001; 292:468-472.

4. Kaelin WG, Jr, Ratcliffe PJ. Oxygen sensing by metazoans: the central role of the HIF hydroxylase pathway. Mol Cell. 2008; 30:393-402.

5. Xia M, Huang R, Sun Y, Semenza GL, Aldred SF, Witt KL, Inglese J, Tice RR, Austin CP. Identification of chemical compounds that induce HIF-1alpha activity. Toxicol Sci. 2009; 112:153-63.

6. Semenza G L. Targeting HIF-1 for cancer therapy. Nat Rev Cancer. 2003; 3:721-732.

7. McCarty MF, Barroso-Aranda J, Contreras F. Practical strategies for suppressing hypoxia-inducible factor activity in cancer therapy. Med Hypotheses. 2010; 74:789-797.

8. Semenza GL. Defining the role of hypoxia-inducible factor 1 in cancer biology and therapeutics. Oncogene. 2010; 29:625-634.

9. Nardinocchi L, Pantisano V, Puca R, Porru M, Aiello A, Grasselli A, Leonetti C, Safran M, Rechavi G, Givol D, Farsetti A, D'Orazi G. Zinc downregulates HIF-1a and inhibits its activity in tumor cells in vitro and in vivo. PLoS ONE. 2010; 5:1-12.

10. Ebos JM, Lee CR, Cruz-Munoz W, Bjarnason GA, Christensen JG, Kerbel RS. Accelerated metastasis after short-term treatment with a potent inhibitor of tumor angiogenesis. Cancer Cell. 2009; 15:232-239.

11. Pàez-Ribes M, Allen E, Hudock J, Takeda T, Okuyama H, Viñals F, Inoue M, Bergers G, Hanahan D, Casanovas O. Antiangiogenic therapy elicits malignant progression of tumors to increased local invasion and distant metastasis. Cancer Cell. 2009; 15:220-231.

12. Li X, Lu Y, Liang $\mathrm{K}$, Pan $\mathrm{T}$, Mendelsohn J, Fan Z. Requirement of hypoxia-inducible factor-1alpha downregulation in mediating the antitumor activity of the antiepidermal growth factor receptor monoclonal antibody cetuximab. Mol Cancer Ther. 2008; 7:1207-1217.

13. Beyersmann D, Haase H. Functions of zinc in signaling, proliferation and differentiation of mammalian cells. Biometals. 2001; 14:331-341.

14. Jackson KA, Valentine RA, Coneyworth LJ, Mathers JC, Ford D. Mechanisms of mammalian zinc-regulated gene expression. Biochem Soc Trans. 2008; 36:1262-1266.

15. Fong LY, Jiang Y, Rawahneh ML, Smalley KJ, Croce CM, Farber JL, Huebner K. Zinc supplementation suppresses 4-nitroquinoline 1-oxide-induced rat oral carcinogenesis. 
Carcinogenesis. 2011; 32:554-560.

16. Ho E. Zinc deficiency, DNA damage and cancer risk. J Nutr Biochem. 2004; 15:572-578.

17. Nardinocchi L, Puca R, Sacchi A, Rechavi G, Givol D, D'Orazi G. Targeting hypoxia in cancer cells by restoring homeodomain interacting protein-kinase 2 and p53 activity and suppressing HIF-1alpha. PLoS ONE. 2009; 4:6819.

18. Puca R, Nardinocchi L, Gal H, Rechavi G, Amariglio N, Domany E, Notterman DA, Scarsella M, Leonetti C, Sacchi A, Blandino G, Givol D, D’Orazi G. Reversible dysfunction of wild-type p53 following homeodomaininteracting protein kinase-2 knockdown. Cancer Res. 2008; 68:3707-3714.

19. Subramanian A, Tamayo P, Mootha VK, Mukherjee S, Ebert BL, Gillette MA, Paulovich A, Pomeroy SL, Golub TR, Lander ES, Mesirov JP. Gene set enrichment analysis: a knowledge-based approach for interpreting genomewide expression profiles. Proc Natl Acad Sci U S A. 2005; 102:15545-15550.

20. Chi JT, Wang Z, Nuyten DS, Rodriguez EH, Schaner ME, Salim A, Wang Y, Kristensen GB, Helland A, BørresenDale AL, Giaccia A, Longaker MT, Hastie T, Yang GP, van de Vijver MJ, Brown PO. Gene expression programs in response to hypoxia: cell type specificity and prognostic significance in human cancers. PLoS Med. 2006; 3:e47.

21. Elvidge GP, Glenny L, Appelhoff RJ, Ratcliffe PJ, Ragoussis J, Gleadle JM. Concordant regulation of gene expression by hypoxia and 2-oxoglutarate-dependent dioxygenase inhibition: the role of HIF-1alpha, HIF-2alpha, and other pathways. J Biol Chem. 2006; 281:15215-15226.

22. Harris AL. Hypoxia--a key regulatory factor in tumour growth. Nat Rev Cancer. 2002; 2:38-47.

23. Jiang Y, Zhang W, Kondo K, Klco JM, St Martin TB, Dufault MR, Madden SL, Kaelin WG Jr, Nacht M. Gene expression profiling in a renal cell carcinoma cell line: dissecting VHL and hypoxia-dependent pathways. Mol Cancer Res. 2003; 1:453-462.

24. Leonard MO, Cottell DC, Godson C, Brady HR, Taylor CT. The role of HIF-1 alpha in transcriptional regulation of the proximal tubular epithelial cell response to hypoxia. J Biol Chem. 2003; 278:40296-40304.

25. Manalo DJ, Rowan A, Lavoie T, Natarajan L, Kelly BD, Ye SQ, Garcia JG, Semenza GL. Transcriptional regulation of vascular endothelial cell responses to hypoxia by HIF-1. Blood. 2005; 105:659-669.

26. Mense SM, Sengupta A, Zhou M, Lan C, Bentsman G, Volsky DJ, Zhang L. Gene expression profiling reveals the profound upregulation of hypoxia-responsive genes in primary human astrocytes. Physiol Genomics. 2006; 25:435-449

27. Mizukami Y, Jo WS, Duerr EM, Gala M, Li J, Zhang X, Zimmer MA, Iliopoulos O, Zukerberg LR, Kohgo Y, Lynch MP, Rueda BR, Chung DC. Induction of interleukin-8 preserves the angiogenic response in HIF-1alpha-deficient colon cancer cells. Nat Med. 2005; 11:992-997.

28. Weinmann M, Belka C, Güner D, Goecke B, Müller I, Bamberg M, Jendrossek V. Array-based comparative gene expression analysis of tumor cells with increased apoptosis resistance after hypoxic selection. Oncogene. 2005; 24:5914-5922.

29. Winter SC, Buffa FM, Silva P, Miller C, Valentine HR, Turley H, Shah KA, Cox GJ, Corbridge RJ, Homer JJ, Musgrove B, Slevin N, Sloan P, Price P, West CM, Harris AL. Relation of a hypoxia metagene derived from head and neck cancer to prognosis of multiple cancers. Cancer Res. 2007; 67:3441-3449.

30. Vengellur A, Phillips JM, Hogenesch JB, LaPres JJ. Gene expression profiling of hypoxia signaling in human hepatocellular carcinoma cells. Physiol Genomics. 2005; 22:308-318.

31. Vengellur A, Woods BG, Ryan HE, Johnson RS, LaPres JJ. Gene expression profiling of the hypoxia signaling pathway in hypoxia-inducible factor 1alpha null mouse embryonic fibroblasts. Gene Expr. 2003; 11:181-197.

32. Bellot G, Garcia-Medina R, Gounon P, Chiche J, Roux D, Pouysségur J, Mazure NM. Hypoxia-induced autophagy is mediated through hypoxia-inducible factor induction of BNIP3 and BNIP3L via their BH3 domains. Mol Cell Biol. 2009; 29:2570-2581.

33. Kaluz S, Kaluzova M, Liao SY, Lerman M, Stanbridge EJ. Transcriptional control of the tumor- and hypoxia-marker carbonic anhydrase 9: A one transcription factor (HIF-1) show? Biochim Biophys Acta. 2009; 1795:162-172.

34. Allikmets R, Gerrard B, Hutchinson A, Dean M. Characterization of the human ABC superfamily: isolation and mapping of 21 new genes using the expressed sequence tags database. Hum Mol Genet. 1996; 5:1649-1655.

35. Benita Y, Kikuchi H, Smith AD, Zhang MQ, Chung DC, Xavier RJ. An integrative genomics approach identifies Hypoxia Inducible Factor-1 (HIF-1)-target genes that form the core response to hypoxia. Nucleic Acids Res. 2009; $37: 4587-4602$.

36. Choi DW, Seo YM, Kim EA, Sung KS, Ahn JW, Park SJ, Lee SR, Choi CY. Ubiquitination and degradation of homeodomain-interacting protein kinase 2 by WD40 repeat/SOCS box protein WSB-1. J Biol Chem. 2008; 283:4682-4689.

37. Puca R, Nardinocchi L, Sacchi A, Rechavi G, Givol D, D'Orazi G. HIPK2 modulates p53 activity towards proapoptotic transcription. Mol Cancer. 2009; 14:8:85.

38. Calzado MA, de la Vega L, Moller A, Bowtell DD, Schmitz ML. An inducible autoregulatory loop between HIPK2 and Siah2 at the apex of the hypoxic response. Nature Cell Biol. 2009; 11:85-91.

39. Price AL, Patterson NJ, Plenge RM, Weinblatt ME, Shadick NA, Reich D. Principal components analysis corrects for stratification in genome-wide association studies. Nature 
Genet. 2006; 38:904-909.

40. Wouters BG, Koritzinsky M. Hypoxia signalling through $\mathrm{mTOR}$ and the unfolded protein response in cancer. Nat Rev Cancer. 2008; 8:851-864.

41. Gatenby RA, Gillies RJ. Why do cancers have high aerobic glycolysis? Nat Rev Cancer. 2004; 11:891-899.

42. Kim JW, Dang CV. Cancer's molecular sweet tooth and the Warburg effect. Cancer Res. 2006; 66:8927-8930.

43. Fulds S, Debatin K-M. HIF-1-regulated glucose metabolism. A key to apoptosis resistance? Cell Cycle. 2007; 6:790-792.

44. Sendoel A, Kohler I, Fellmann C, Lowe SW, Hengsrtner MO. HIF-1 antagonizes p53-mediated apoptosis through a secreted neuronal tyrosinase. Nature. 2010; 465:577-583.

45. Nardinocchi L, Puca R, D’Orazi G. HIF-1a antagonizes p53-mediated apoptosis by triggering HIPK2 degradation. Aging (Albany NY). 2011; 3:33-43.

46. Puca R, Nardinocchi L, Givol D, D’Orazi G. Regulation of p53 activity by HIPK2: molecular mechanisms and therapeutical implications in human cancer cells Oncogene. 2010; 29:4378-4387.

47. Nardinocchi L, Puca R, Givol D, D’Orazi G. Counteracting MDM2-induced HIPK2 downregulation restores HIPK2/ p53 apoptotic signaling in cancer cells. FEBS Lett. 2010; 584:4253-4258.

48. Affymetrix. Guide to probe logarithmic intensity error (PLIER) estimation. available at http://www.affymetrix. com/support/technical/technotes/plier_technote.pdf. 2005.

49. Ballman KV, Grill DE, Oberg AL, Therneau TM. Faster cyclic loess: normalizing RNA arrays via linear models. Bioinformatics. 2004; 20:2778-2786.

50. Zeisel A, Amir A, Kostler WJ, Domany E. Intensity dependent estimation of noise in microarrays improves detection of differentially expressed genes. BMC Bioinformatics. 2010; 11:400-407.

51. Tsafrir D, Tsafrir I, Ein-Dor L, Zuk O, Notterman DA, Domany E. Sorting points into neighborhoods (SPIN): data analysis and visualization by ordering distance matrices. Bioinformatics. 2005; 21:2301-2308. 